\section{A new understanding of endurance exercise}

\author{
Joseph Sepe, ${ }^{1}$ Raffaella D'Alessandro² \\ 'Life Sciences, University of Maryland \\ University College, Adelphi, USA; \\ 2Department of Cardiothoracic and \\ Respiratory Sciences, Monaldi Hospital, \\ Naples, Italy
}

Longevity gained through regular exercise is cemented into popular wisdom. Today the cardiovascular benefits from moderate exercise are undeniable and the data from large cohort studies keep piling up., ${ }^{1,2}$ But the benefits from endurance exercise are less clear and even less is known about its effects on longevity. ${ }^{3}$ What endurance exercise cannot do is explain away myocardial remodeling ${ }^{4}$ and arrhythmias. $^{5}$

Despite this, until now no underlying mechanism to explain increased life expectancy resulting from endurance exercise has been very convincing. Harman suggested in a groundbreaking paper that mitochondria are the orchestrators of aging. ${ }^{6} \mathrm{He}$ argued that mitochondria are both producing and being damaged by free radicals, but antioxidants do not enter the mitochondria.

Reactive oxygen species are routinely inactivated to relieve oxidative stress by the enzymes glutathione peroxidase, superoxide dismutase and catalase. ${ }^{7}$ We know something about cellular system deficiencies of these enzymes in mice and men. They lead to endothelial dysfunction ${ }^{8}$ and increased risk of cardiovascular events ${ }^{9}$ respectively.

On the other hand, exogenous antioxidants seem to play no role and this avenue of research is fraught with controversy. ${ }^{10} \mathrm{In}$ fact, exogenous antioxidants have even been shown to be harmful. In the AlphaTocopherol/Beta-Carotene Cancer Prevention Study, there was an 18 percent higher incidence of lung cancer among men who received beta-carotene than among those who did not and increased incidence of cardiac death, hemorrhagic stroke, and ischemic heart disease. $^{11}$

With particular regard to the effects of exercise on mitochondria and heart, the role of mitochondrial biogenesis under physiological and pathological states has to be considered. Conditions requiring increased cardiac workload promote increasing heart mass and changes in genetic expression; the variations in response to endurance training include alterations in mitochondrial biogenesis (and the same event has also been observed in heart failure),${ }^{12}$ in mitochondrial oxidative stress molecules, in mitochondrial antioxidant enzymes $^{13}$ resulting in a total cardioprotective effect. $^{14}$

Some light has recently been shed on the mitochondrial role of aging using mouse models for progenoid aging and this may set a capstone on Harman's lifelong work.

In a pioneering study, Safdar used the mtDNA mutator mouse to find out whether endurance exercise can nullify multisystem degeneration and premature aging in these animals. ${ }^{15}$ Such mice provide a model for mtDNA mutations that accumulate during natural aging and result in electron transport alterations leading to increased activation of apoptosis. ${ }^{16,17}$

\section{How was the study done?}

In Safdar's study, one group of mice were kept sedentary and another group from agematched littermates was forced to run on a wheel for 45 min three times a week.

\section{What did they find?}

As expected, sedentary mice displayed signs of accelerated aging: alopecia, graying hair, weight loss, poor body condition, and impaired mobility. By contrast, In the exercising group, five months of endurance exercise induced systemic mitochondrial biogenesis, prevented mtDNA depletion and mutations, increased oxidative capacity and respiratory chain assembly, restored mitochondrial morphology, and blunted apoptosis. These adaptations conferred complete phenotypic protection, maintained weight reduced multisystem pathology and prevented premature mortality.

\section{What were the limitations}

\section{of the study?}

The study probably does not provide argument for the general role of mitochondria but rather for the role in this specific mouse model. These results must be interpreted with caution because of the small number of animals ${ }^{10}$ in each group.

\section{What are the implications}

\section{of the study for cardiogenetics?}

In light of the maternal pattern of mitochondrial inheritance, the role of the mitochondrial genome must be considered with respect to human genetic disease.

The mitochondrial genome has a high mutation rate. The overall mtDNA mutation rate per base pair per fly generation in Drosophila is estimated to be about 10 -fold higher than the nuclear mutation rate, but the mitochondrial major strand $\mathrm{G}>\mathrm{A}$ mutation rate is about 70 fold higher than the nuclear rate. ${ }^{18}$ Mitochondrial mutations have been associated with heart failure, heart block, and cardiomyopathy. ${ }^{19}$ Mitochondrial myopathies can arise from deletions in mitochondrial DNA or mito-
Correspondence: Joseph Sepe, University of Maryland University College, 3501 University Boulevard East, Adelphi, MD 20783-8085, USA.

E-mail: jsepe@faculty.ed.umuc.edu

Key words: education, cardiogenetics, endurance exercise, cardiovascular benefits.

Received for publication: 3 November 2011.

Revision received: 3 November 2011.

Accepted for publication: 24 November 2011.

This work is licensed under a Creative Commons Attribution NonCommercial 3.0 License (CC BYNC 3.0).

(C) Copyright J. Sepe and R. D'Alessandro et al., 2011 Licensee PAGEPress, Italy

Cardiogenetics 2011; 1:e14

doi:10.4081/cardiogenetics.2011.e14

chondrial proteins associated with ATP electron transport chain enzyme defects that alter mitochondrial morphology. ${ }^{20}$ Other mitochondrial myopathies affect ATP production abnormalities of fatty acid oxidation (acyl CoA dehydrogenase deficiencies) and carnitine deficiency, as well as infiltrative myopathies, i.e., glycogen storage diseases (type II; autosomal recessive Pompe disease), Hunter's and Hurler's diseases, and the transient and nonfamilial cardiomyopathy as part of generalized organomegaly. ${ }^{21}$

Oxidant stress plays some role in the development and progression of cardiovascular disease because animal models and humans with antioxidant deficiencies seem to be subject to greater injury and dysfunction. What role does mitochondrial DNA (mtDNA) mutagenesis have in human aging? Is there any evidence? Is there a central mechanism driving human aging and associated pathologies? Could it be linked to mtDNA mutagenesis and depletion, enhanced systemic apoptosis, or some other form of mitochondrial dysfunction?

The results found in the study published by Safdar and coworkers can be contextualized in the current knowledge about the involvement of mitochondria in cardiac changes due both to physiologic and pathologic conditions.

The role of mitochondria in cardioprotection has been suggested to be due to the effect of exercise on the reduction of the so-called cardiac mitotoxicity, a condition particularly frequent in association with aging, diabetes, administration of anti-cancer agents and ischemia-reperfusion. ${ }^{22}$

The involvement of mitochondria in the changes of heart mediated by endurance training is confirmed also by morphological variations affecting those organelles. Exercise training leads enhance in stroke volume and cardiac output, features od cardiac enlargement; experience with other conditions, such as copper 
deficiency, demonstrates that heart enlargement can be due to an increase in mitochondria number or in the presence of megamitochondria, underlining the role of mitochondria in the responses to endurance exercise. By a substructural morphological point of view, studies on rats indicate that the enlargement induced by endurance training leads to a disorganization and a number reduction of the internal cristae of giant mitochondria and mitochondrial matrix density results partially lost. ${ }^{23}$

The role of exercise on preventing cardiac dysfunction has been discussed in the past and different mechanisms have been proposed to explain the effects on cells and, especially, on cardiomyocytes. Exercise promotes the function of defense systems by inducing redox changes (inducible antioxidants) and myocardial heat shock proteins, improving cardiac antioxidant capacity and increasing cardioprotective molecules: events that seem to lead to an overall cardioprotective effect. ${ }^{14}$

Recent studies propose that exercise training has a positive effect on both cardiac subsarcolemmal and intermyofibrillar mitochondria. Experiments comparing heart tissues extracted from sedentary rats and trained rats demonstrate that the levels of mitochondrial oxidative stress molecules (such as 4-hydroxynonenal-conjugated proteins) are increased in the first group, while the levels of mitochondrial antioxidant enzymes (such as copperzinc superoxide dismutase, manganese superoxide dismutase, and glutathione peroxidase) are increased in the second group. ${ }^{13}$

Endurance exercise is reported also to inhibit apoptosis reducing caspase- 3 and -9 activities and $\mathrm{Bax} / \mathrm{Bcl}-2$ ratio (found increased in hyperglycemic sedentary groups of rats). This modulation in apoptosis processes, accompanied by control of known pore regulatory/component proteins (such as ANT and Cyp D), seems to be beneficial for cardiac tissue particularly in clinical conditions in which exercise training has been demonstrated to be protective, as in the case of diabetic cardiomyopathy. ${ }^{24}$

Genetic expression of specific transcription factors has been suggested as a factor involved in the adaption of cardiac tissue to changes in the environmental conditions. Mitochondrial biogenesis regulatory proteins, such as PGC$1 \alpha$ PGC-1 $\beta$ and are particularly expressed in mitochondria-rich tissues and are both involved in the control of mitochondrial biogenesis. A specific role in the response to exercise, starvation and cold has been demonstrated for the first one, which then represents a critical molecule involved in the control of cardiac mitochondrial number and function whose expression is dependent on specific energy demands. PGC-1 $\beta$ has been suggested to be effective in constitutive mitochondrial biogenesis. $^{12}$
Since development and disease states result from the interaction of genes and environment, lifestyle decisions made early in life may have profound and long-reaching effects. This remains to be demonstrated in individuals predisposed to cardiovascular disease.

\section{Future considerations}

Intrigued by our growing awareness of cell biology and the impact of lifestyle choices on heart disease, clinical studies should soon spawn on prevention.

\section{Conclusions}

The human body is made up of billions of cells that grow, divide, and then die in a predictable manner. Harman proposed a cellular mechanism to explain, at least some of the aging process - Safdar showed its relevance in living models. We need to bridge this with randomized, double-blind, controlled, large cohort human studies in cardiology, which are the clinical gold standard.

\section{References}

1. Guillum RF, Mussolino ME, Ingram DD. Physical activity and stroke incidence in women and men. The NHANES I epidemiologic follow-up study. Am J Epidemiol 1996;143:860-9.

2. Blair SN. Physical inactivity: the biggest public health problem of the 21st century. Br J Sports Med 2009;43:1-2.

3. Ruiz J, Morán M, Arenas J, Alejandro L. Strenuous exercise improves life expectancy: it's in our genes Br J Sports Med 2011;45:159-61.

4. Volders PG. Marathon rat: myocardial remodeling in an animal model of vigorous endurance exercise and implications for humans. Circulation 2011;123:5-7.

5. Heidbüchel J, Hoogsteen J, Fagard R, et al. High prevalence of right ventricular involvement in endurance athletes with ventricular arrhythmias. Role of an electrophysiologic study in risk stratification. Eur Heart J 2003;24:1473-80.

6. Harman D. The biologic clock: the mitochondria? J Am Geriatr Soc 1972;20:145-7.

7. Forsberg L, de Faire U, Morgenstern R. Oxidative stress, human genetic variation, and disease. Arch Biochem Biophys 2001;389:84-93.

8. Forgione MA, Cap A, Liao R, et al. Heterozygous cellular glutathione peroxi- dase deficiency in the mouse: abnormalities in vascular and cardiac function and structure. Circulation 2002;106:1154-8.

9. Blankenberg S, Rupprecht HJ, Bickel C, et al. Glutathione Peroxidase 1 Activity and Cardiovascular Events in Patients with Coronary Artery Disease. N Engl J Med 2003;349:1605-13.

10. Howes R. The free radical fantasy: a panoply of paradoxes. Ann N Y Acad Sci 2006; 1067:22-6.

11. Heinonen OP, Huttunen JK, Albanes D, et al. The effect of vitamin $\mathrm{E}$ and beta carotene on the incidence of lung cancer and other cancers in male smokers. The Alpha-Tocopherol, Beta Carotene Cancer Prevention Study Group. N Engl J Med 1994;330:1029-35.

12. Rimbaud S, Garnier A, Ventura-Clapier R. Mitochondrial biogenesis in cardiac pathophysiology. Pharmacol Rep 2009;61: 131-8.

13. Lee Y, Min K, Talbert EE, et al. Exercise Protects Cardiac Mitochondria Against Ischemia-Reperfusion Injury. Med Sci Sports Exerc 2011. [Epub ahead of print].

14. Ascensão A, Ferreira R, Magalhães J. Exercise-induced cardioprotection--biochemical, morphological and functional evidence in whole tissue and isolated mitochondria. Int $\mathrm{J}$ Cardiol 2007;117:1630.

15. Safdar A, Bourgeois JM, Ogborn DI, et al. Endurance exercise rescues progeroid aging and induces systemic mitochondrial rejuvenation in mtDNA mutator mice. Proc Natl Acad Sci U S A 2011;108:4135-40.

16. Edgar D, Trifunovic A. The mtDNA mutator mouse: Dissecting mitochondrial involvement in aging. Aging (Albany NY) 2009;1: 1028-32.

17. Hiona A, Sanz A, Kujoth GC, et al. Mitochondrial DNA mutations induce mitochondrial dysfunction, apoptosis and sarcopenia in skeletal muscle of mitochondrial DNA mutator mice. PloS One 2010;5: e11468.

18. Haag-Liautard C, Coffey N, Houle D, et al. Direct estimation of the mitochondrial DNA mutation rate in Drosophila melanogaster. PloS One 2008;6:e204.

19. Holmgren D, Wåhlander H, Eriksson BO, et al. Cardiomyopathy in children with mitochondrial disease. Eur Heart J 2003;24: 280-8.

20. Ponzetto N, Bresolin A, Bordoni M, et al. Kearns-Sayre syndrome: Different amounts of deleted mitochondrial DNA are present in several autoptic tissues C. J Neurol Sci 1990;96;207-10.

21. Berardo A, DiMauro S, Hirano M. A Diagnostic Algorithm for Metabolic Myopathies. Curr Neurol Neurosci Rep 2010; 10:118-26. 
22. Ascensão A, Lumini-Oliveira J, Oliveira PJ, Magalhães J. Mitochondria as a target for exercise-induced cardioprotection. Curr Drug Targets 2011;12:860-71.

23. Hoppel CL, Tandler B, Fujioka H, Riva A.
Dynamic organization of mitochondria in human heart and in myocardial disease. Int J Biochem Cell Biol 2009;41:1949-56.

24. Lumini-Oliveira J, Magalhães J, Pereira $\mathrm{CV}$, et al. Endurance training reverts heart mitochondrial dysfunction, permeability transition and apoptotic signaling in longterm severe hyperglycemia. Mitochondrion 2011;11:54-63. 